\title{
Prevención de la infección asociada a catéteres: utilidad y costo-eficacia de los catéteres con antisépticos en pediatría
}

\author{
ANA M. LENZ ${ }^{1}$, JUAN C. VASSALLO ${ }^{1}$, GUILLERMO E. MORENO ${ }^{1}$, MARÍA ALTHABE ${ }^{1}$, \\ SILVIA GÓMEZ ${ }^{1}$, RICARDO MAGLIOLA ${ }^{1}$, LIDIA CASIMIR ${ }^{2}$, ROSA BOLOGNA ${ }^{3}$, \\ JORGE BARRETTA ${ }^{4}$, PABLO RUFFA ${ }^{4}$ \\ 1. $\mathrm{UCl} 35$ \\ 2. Microbiología \\ 3. Infectología. \\ 4. Cirugía cardiovascular \\ Hospital Nacional de Pediatría "Prof. Dr. Juan P. Garrahan". Buenos Aires, Argentina.
}

\begin{abstract}
Prevention of catheter-related infection; usefulness and cost-effectiveness of antiseptic catheters in children
\end{abstract}

Objective: To evaluate the cost-effectiveness of the antiseptic-impregnated catheter compared with conventional catheters in preventing catheter-related blood stream infections (CR-BSI). Design: Cost-effectiveness analysis; clinical trial, experimental, randomized, controlled, prospective, open label. Patients and Methods: A 172 patient cohort, under 1-year-old or less than $10 \mathrm{~kg}$, postoperative cardiovascular children with central venous catheters (CVC) admitted to Cardiac Intensive Care Unit (UCI 35) at Hospital Nacional de Pediatría “Prof. Dr. Juan P. Garrahan”, since September 2005 to December 2007. Demographic and CVC data were retrieved to compare: age, gender, weight, diagnosis, surgery, CVC days, costs and complications. Intervention: CVC Arrow, double-lumen, $>48 \mathrm{~h}$ of duration; intervention group: antiseptic-impregnated CVC vs control group: CVC without antiseptics (conventional). Results: The incidence of CR-IE (CR-Infected Events: colonization, local infection and/or CRBSI; combined end point) was $27 \%$ for antiseptic-impregnated CVC vs. $31 \%$ for conventional catheters $(\mathrm{p}=0.6)$ with similar accumulated incidence of CR- BSI: 2.8 vs. 3.3 per 1000 days catheter. We found no differences between groups, except in weight: median $4.0 \mathrm{~kg}$ (r 2-17) vs $4.7 \mathrm{~kg}$ (r 2-9) p $=0.0002$ and age, median 2 months ( $r 1$ - 48) vs 5 months ( $r 1$ - 24) p= 0.0019 in antiseptic-impregnated CVC group. These differences, though statistically significant were clinically non relevant. Median cost per patient during intensive care stay in the conventional CVC group was \$3.417 (359-9.453) and in the antisepticimpregnated-CVC group was $\$ 4.962$ (239-24.532), $\mathrm{p}=0.10$. Conclusions: The use of antiseptic-impregnated CVC compared with conventional CVC did not decrease CR-BSI in this population. The cost per patients was higher in the antiseptic impregnated CVC group. These results do not support the routine use of this type of CVC in our population.

(Key words: Central venous catheter, catheter-related blood stream infection, antiseptic-impregnated catheter, cost-effectiveness).

Arch Argent Pediatr 2010; 108 (3): 209-215 


\section{RESUMEN}

Objetivo: Evaluar la costo-eficacia de catéteres venosos centrales con antisépticos comparados con catéteres convencionales en la prevención de episodios infecciosos asociados. Diseño: Análisis de costo-eficacia; ensayo clínico, experimental, aleatorizado, controlado, abierto. Población y Métodos: Pacientes con catéteres venosos centrales, menores de 1 año o $10 \mathrm{~kg}$, posquirúrgicos cardiovasculares. Se compararon las características de los pacientes y la permanencia, costos y complicaciones de los catéteres venosos centrales. Intervención: Catéter venoso central Arrow ${ }^{\circledR}$, doble lumen, > 48 h de duración; grupo de intervención: catéteres con antiséptico contra grupo control: catéteres convencionales. Resultados: Se estudiaron 172 catéteres pertenecientes a 149 pacientes. La tasa cruda de episodios infecciosos asociados fue del 27\% en los catéteres con antisépticos y $31 \%$ en los catéteres convencionales ( $\mathrm{p}=0,6)$, con incidencia acumulada similar de bacteriemia asociada a catéter: 2,8 contra 3,3 por 1000 días-catéter. No se encontraron diferencias entre los grupos, excepto en el menor peso: mediana 4,0 kg (r 2-17) contra 4,7 kg (r2-9) p = 0,0002 y edad mediana $2 \mathrm{~m}$ (r 1-48) contra 5 m (r 1-24) $\mathrm{p}=0,0019$ en la población de catéteres venosos centrales con antisépticos. Estas diferencias se consideraron clínicamente irrelevantes. El costo promedio por paciente internado en el grupo con catéter convencional fue \$ 3.417 (359-9.453) y en el grupo catéter con antisépticos fue de \$ 4.962 (239-24.532), p= 0,10. Conclusiones: El uso de catéteres venosos centrales con antisépticos comparado con el de catéteres convencionales no redujo los episodios infecciosos asociados, con una tendencia de mayor costo en el grupo de catéteres con antisépticos. Estos resultados no respaldan el uso de estos catéteres en nuestro medio.

(Palabras clave: Catéter venoso central, infección asociada a catéter, catéter impregnado con antisépticos, costo-eficacia).

Arch Argent Pediatr 2010; 108 (3): 209-215

\section{ESTE TRABAJO LO PUEDE ENCONTRAR EN EXTENSO EN WWW.SciELO.ORG}

